\title{
Contents, Vol. 40, 1918
}

\section{Inhalts - Verzeichnis.}

Seite

Original-Arbeiten.

Blaskovics, L., Über Totalplastik des unteren hides. Bildung einer hinteren Lidplatte durch

Transplantation ein $i$ s Tarsus- und Bindehautstreifens aus dem Obsrlide . . . 222

Cramer, E.\} Ersatz der Unterlidbildung durch Hebung und

Verlagerung des Augenhöhleninhalts nach vorn ....151

Esser, A., Schattenbildung in der normalen Linse .... 232

Fürstenau, E., Einwirkung des Trypaflavins auf augenpatho-

gene Keime 1

Hensen, H., Ein Fall von Sehnervenatrophie durch Band-

wurmtoxine 331

Heßberg, R., Über die Verwendung des Krysolgan bei fcuber-

kulösen Augenerkrankungen 324

Horlacher, J., Das Verhalten der menschlichen Linse in Bezügauf die Form von Alterstrübungen bei 166 Personen haAlter von 51-81 Jahren 33

Koeppe, L., Über derzeitigen Stand der Glaukomforschung ander Gullstrandschen

Nernstspaltlampe sowde den weiterenAusbau der Glaukom-Frühdiagnose vermittelst dieserUntersuchungsmethode 138

-,- Entgegnung auf die Arbeit Levinsohns: Zur Entstehungdes Glaukoms durch

Pigmentinfiltration der vordexenAbflußwege des Auges 349

Levinsohn, Zur Entstehung des Glaukoms durch Pigment-infiltration der vorderen Abflußwege des Auges .... 344

Pflugk, A. v, u. M. v. Bohr, Beiträge zur Entwicklung der

Kenntnis von der Brille $\quad 50$

Pichl\&r + A., Zur Technik der magnetischen Splitterausziehung 30

-,- Simuliertes Schielen 157

-,- Fälle von akuter.. rasch heilender, beiderseitiger Abdu-

zenslähmang, wahrscheinlich durch Influenza bedingt . 334

- ,- Erfahrungen über die Koch-Weeks-Konj unktivitis im

Kriege, mit einem Anhang über Diplobazilluskatarrh . 337

Polyåk. L., Die Heilung der Tuberkulose des Tränensackes

auf intranasalem $\mathrm{W}_{\text {ige }}$

181

Salus, R.. Zur Frage nach der Entstehung des Altersstars . 23

- iv -

Seite

Schumacher, G, t Histologische Untersuehung der Sternfigur der Macula bei Stauungspapille an Gefrierschnitten.

(Hierzu Tafel I) 305 
Schoute, G. J.. Gürtelförmige Hornhauttrübung

Stargardt, H., Über die Brauchbarkeit von Radiu«üeuchtfarben

für Adaptometer 228

-,- Naht des durchrissenen unteren Tränenkanälchens . . 320 Vogt, A., Der Altersstar, seine

Heredität und seine Stellung

zu exogener Krankheit und Senna m 123

-.- Klinische und experimentelie Untersuchungen über

die Genese der Foss ${ }_{i} \cdot 1 / 8$ sschen Ringtrübung 204

Wirths, M., Über Linsentrübungen bei Anwesenheit von

Kupfer im Angeninnern $\quad 164$

Wolff, K., Mannschaftsuntersuehungen mit von $\mathrm{j}^{7} / 80^{7} / 8$ rschen

Femrohrbrillen (C. Zeiß) 232

Zade, Beitrag zur Kenntnis des Exophthalmus bei Neben-

höhlen-Erkrankung 154

Berichte über die deutsche ophthalmologische Literatar.

Sympathisehe Ophthalmic Von Prof. Dr. C. H. Battler in

Königsberg 169

Spezielle Pathologic und Therapie (11. Sem, 1916). Von Dr.

Konrad Schroder in Gera 253

-,- (Jahrgatog 1917). Von Dr. Konrad Schrader in Gera 287

Untersuehungsmethoden (1. u. II. Sem. 1917). Von Dr. Bichard

Kramer in Wien 294

Berichte über die ausländische ophthalmologische Literatur.

Bericht über die holländische ophthalmologische Literatur desJahres 1918 (II. Hälfte). Von Prof.

Dr. W. Koster Gzn. inHaag und Dr. G. J.'Schoute in Amsterdam 350

Bericht über die 41. Zusammenkunft der Ophthalmologischen Gesellschaft in Heidelberg am 5.

August 1918. - Unter Benutzung von Selbstberichten von Prof. B. Cords in Bonn 78

Therapeutische Omschau 172

Diagnostische Notizen 173

Buchanzeigen 117, 173

y. yteratur-Verzeichnis $\quad 119,179$

302

Personalien 302 